\title{
The Schur-Szegö Composition of Real Polynomials of Degree 2
}

\author{
Soliman AlkhatiB and Vladimir Petrov Kostov \\ Université de Nice \\ Laboratoire de Mathématiques \\ Parc Valrose \\ 06108 Nice Cedex 2 - France \\ khatib@math.unice.fr kostov@math.unice.fr
}

Received: April 16, 2007

Accepted: September 17, 2007

\begin{abstract}
A real polynomial $P$ in one real variable is hyperbolic if its roots are all real. The composition of Schur-Szegö of the polynomials $P=\sum_{j=0}^{n} C_{n}^{j} a_{j} x^{j}$ and $Q=\sum_{j=0}^{n} C_{n}^{j} b_{j} x^{j}$ is the polynomial $P * Q=\sum_{j=0}^{n} C_{n}^{j} a_{j} b_{j} x^{j}$. In the present paper we show how for $n=2$ and when $P$ and $Q$ are real or hyperbolic the roots of $P * Q$ depend on the roots or the coefficients of $P$ and $Q$. We consider also the case when $n \geq 2$ is arbitrary and $P$ and $Q$ are of the form $(x-1)^{n-1}(x+b)$. This case is interesting in the context of the possibility to present every polynomial having one of its roots at $(-1)$ as a composition of $n-1$ polynomials of the form $(x+1)^{n-1}(x+b)$.
\end{abstract}

Key words: composition of Schur-Szegö, hyperbolic polynomial.

2000 Mathematics Subject Classification: 12D10.

\section{Introduction}

Definition. The composition of Schur-Szegö (CSS) of the two degree $n$ polynomials $P=\sum_{j=0}^{n} C_{n}^{j} a_{j} x^{j}$ and $Q=\sum_{j=0}^{n} C_{n}^{j} b_{j} x^{j}$ (notice their not quite usual form) is the polynomial $P * Q=\sum_{j=0}^{n} C_{n}^{j} a_{j} b_{j} x^{j}$.

Research partially supported by research project 20682 for cooperation between CNRS and FAPESP "Zeros of algebraic polynomials." 
The above definition applies to any complex polynomials. General properties of the CSS can be found in [4]. In particular, the Szegö theorem which says that if all roots of $P$ belong to a circular domain $\mathcal{K}$ (i.e., to the image of the closed unit disc under a fractionally-linear map), then every root of $P * Q$ is of the form $(-\xi \eta)$ where $\xi$ is a root of $Q$ and $\eta \in \mathcal{K}$. Thus the CSS can be used to understand how the roots of a given polynomial are located. (If one can represent a polynomial $R$ as a composition of two polynomials which play the roles of $P$ and $Q$, i.e., the location of whose roots is known, then one can obtain nontrivial information about the location of the roots of $R$.)

In the present paper we consider the case when the polynomials are real. Of particular interest is the subcase when they are hyperbolic, i.e., with all roots real. In this case one can deduce from the Szegö theorem that if all roots of $Q$ have the same sign, then $P * Q$ is hyperbolic and its roots belong to the interval $[-M,-m]$ where $m$ and $M$ are the minimal and maximal pairwise products of roots of $P$ and $Q$ (see [5, Theorem 5.5.5 and Corollary 5.5.10]).

The finite sequence $\alpha:=\left(a_{0}, a_{1}, \ldots, a_{n}\right)$ (defined after the polynomial $P$ ) can be considered as an operator $T_{P}$ in the space of polynomials of degree $n$ acting diagonally in the standard monomial basis according to the formula $T_{P}\left(x^{i}\right)=a_{i} x^{i}$. We call $\alpha$ a finite multiplier sequence of length $(n+1)(\operatorname{FMS}(n+1))$ if for any hyperbolic polynomial $Q$ the polynomial $P * Q$ is also hyperbolic. For the infinite-dimensional analog of an $\operatorname{FMS}(n+1)$ (called a multiplier sequence) see [2, Definition 1.4 and Theorem 3.3].

It turns out that $\alpha$ is an $\operatorname{FMS}(n+1)$ if and only if $P$ is hyperbolic and all nonzero roots of $P$ are of the same sign, see [1, Theorem 3.7]. This nontrivial fact is the reason why in [3] the case of hyperbolic polynomials $P$ and $Q$ is considered one of which has all roots positive or all roots negative. It is shown there (see Proposition 1.4 and Theorem 1.6) that the multiplicities of the roots of $P * Q$ are completely defined by the ones of $P$ and $Q$. In particular, Proposition 1.4 says that if $x_{P}, x_{Q}$ are roots of $P, Q$ of multiplicity respectively $m_{P}, m_{Q}$ where $m_{P}+m_{Q} \geq n$, then $\left(-x_{P} x_{Q}\right)$ is an $\left(m_{P}+m_{Q}-n\right)$-fold root of $P * Q$.

These interesting properties in the case when $P$ and $Q$ are hyperbolic motivate the authors' desire to understand (at least in low degrees) the behavior of the roots of $P * Q$ when the ones of $P$ and $Q$ change. In the present paper we consider in detail the case of real and the case of hyperbolic polynomials for $n=2$, see section 1 . In these cases it is possible to show easily on figures how the roots of $P * Q$ depend on the coefficients or on the roots of $P$ and $Q$. In section 1 we consider also (for any $n \in \mathbf{N}^{*}$ ) a factorization connected with CSS and (in view of this factorization) the composition of two polynomials of the form $(x-1)^{n-1}(x+b)$.

We take into account not only the presence of multiple roots, but also whether the real roots are positive, negative or zero, and whether the real parts of the roots of a conjugate couple are equal to 0 or to the third root. Such information is important when a polynomial is the characteristic polynomial of an ordinary linear differential equation and one considers the problem of stability or which of its solutions has the 
greatest growth rate.

Throughout the paper the polynomials are monic. The following lemma (which is true for any complex polynomials and which can be checked straightforwardly) is used to normalize some roots or coefficients and thus to decrease the number of parameters:

Lemma A. For any $\alpha, \beta \in \mathbf{C}$ one has $P(\alpha x) * Q(\beta x)=(P * Q)(\alpha \beta x)$.

\section{The case $n=2$}

In subsection 1.1 we consider the case when the two polynomials (of degree 2) $P$ and $Q$ are hyperbolic; the parameters are their roots. In subsection 1.3 we consider a generalization of this case for any $n \geq 2$, namely, when the polynomials are of the form $(x-1)^{n-1}(x+b)$. Composition of polynomials of the form $(x+1)^{n-1}(x+b)$ (the sign of 1 is changed for convenience, for purely technical reasons) takes place when one considers a factorization connected with CSS, see subsection 1.2. Some of the proofs of results from subsection 1.1 are postponed till subsection 1.3. In subsection 1.4 we consider the case when the degree 2 polynomials $P$ and $Q$ are real; the parameters are their coefficients.

Finally, in subsection 1.5 we consider the "mixed" situation when one of the polynomials $P$ and $Q$ is real and parameterized by its coefficients while the other is hyperbolic and parameterized by its roots. Such a situation might seem at first glance unnatural to consider, yet in applications, when solving a concrete problem in which the two polynomials play different roles, it might be convenient to have one of them hyperbolic and the other one real.

In the case $n=2$ one can normalize one of the roots or one of the nonzero coefficients of each polynomial to be equal to 1 thanks to Lemma A. This allows only two parameters to be used in the description.

\subsection{The case of hyperbolic polynomials}

Consider for $n=2$ the case when the two polynomials are hyperbolic and neither of them has a double zero root. (In the latter case one has $P * Q=x^{2}$.) In this case one can use Lemma $\mathrm{A}$ and assume that each of them has a root equal to 1, i.e., they are of the form

$$
\begin{aligned}
& P=(x-1)(x+b)=x^{2}+(b-1) x-b, \\
& Q=(x-1)(x+c)=x^{2}+(c-1) x-c
\end{aligned}
$$

where $b, c \in \mathbf{R}$. Hence,

$$
P * Q=x^{2}+\frac{(b-1)(c-1) x}{2}+b c .
$$

The roots of $P * Q$ depend on the parameters $b$ and $c$ where $(-b)$ and $(-c)$ are the second roots of $P$ and $Q$. This polynomial has a double root if and only if the following 


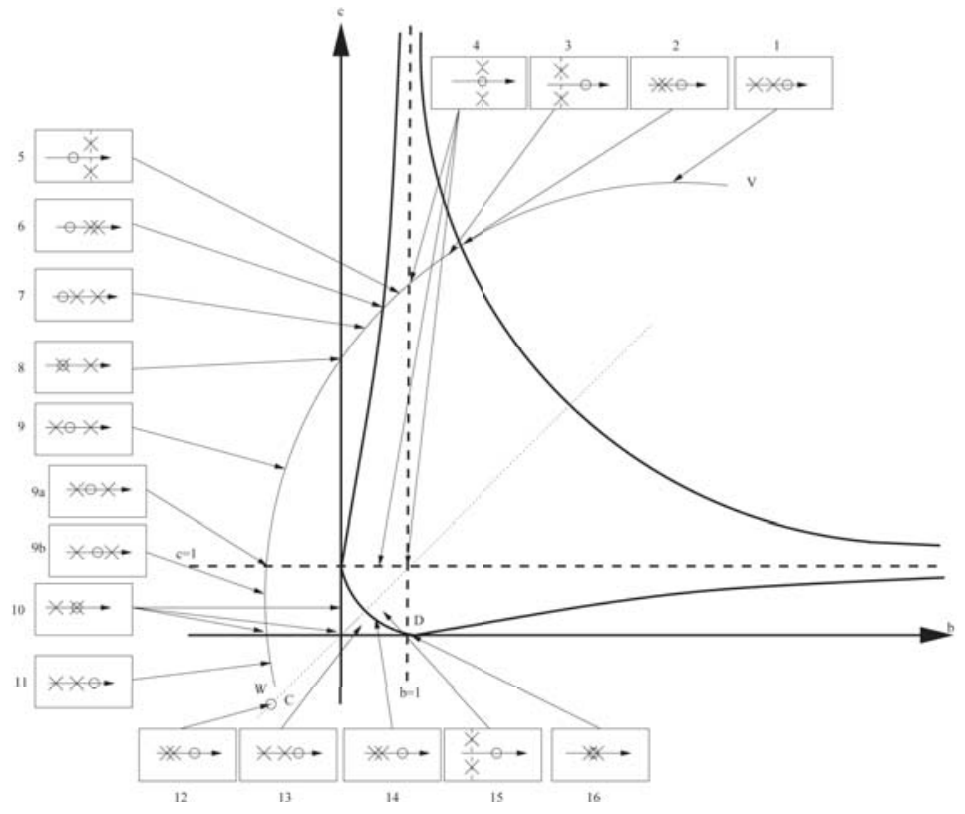

Figure 1 - The curve $\Delta$.

condition holds:

$$
\Delta:(b-1)^{2}(c-1)^{2}-16 b c=0
$$

We present the curve $\Delta$ on figure 1. It is symmetric w.r.t. the bisector $\{b=c\}$ and has two branches. It has also two asymptotes, $\{b=1\}$ and $\{c=1\}$, presented by dashed lines. One of the branches has ordinary tangencies with the coordinate axes at the points $(0,1)$ and $(1,0)$. The curve has an isolated point at $(-1,-1)$ (denoted by $C$ on figure 1 ) which is a singular point (an ordinary double point) of $\Delta$ considered as a complex curve.

The curve $\Delta$ is a particular case of the curve $\Delta_{n}$ drawn on figure 3 in subsection 1.3. (One has $\Delta=\Delta_{2}$.) Therefore we postpone the justification of the description of $\Delta$ till we introduce figure 3. However, most of the properties of $\Delta$ follow from its parameterization, namely,

$$
b(t)=\frac{(t+8)^{2}}{(t-2)^{2}}, \quad c(t)=\frac{(t+3)^{2}}{25}, \quad t \in \mathbf{R} .
$$

The latter does not cover the isolated point $(-1,-1)$. We give the table of variations, see figure 2 .

On figure 1 we show also how the roots of $P * Q$ depend on the parameters $b, c$. These roots are represented in the small rectangles by two multiplication signs. The 


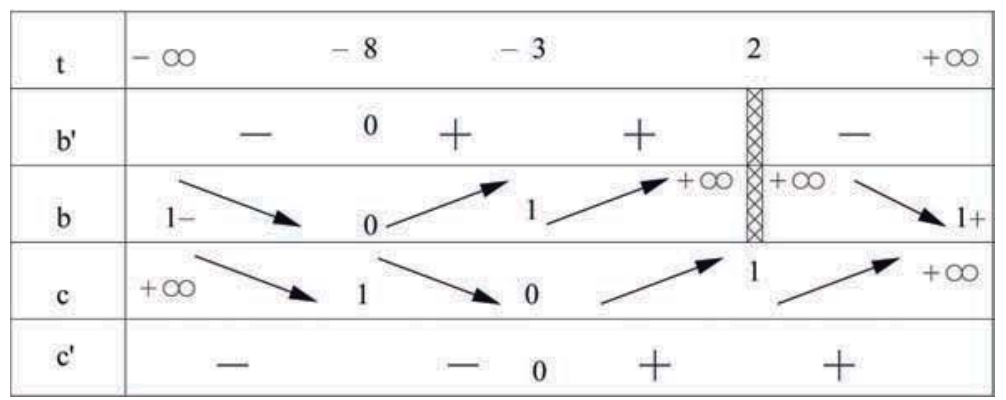

Figure 2 - The table of variations of the curve $\Delta$.

point 0 is represented by a small circle.

When the multiplication signs are very close to one another, this means that $P * Q$ has a double root. Such is the case in rectangles 2, 12, 14 (a double negative root); 6 (a double positive root); 16 (a double zero root). In rectangle 12 one has $b=c=-1$ (this is the isolated point of $\Delta$ ) and the double root equals $(-1)$. In the plane $O b c$ this point is surrounded by points defining polynomials with two real distinct roots.

In rectangles 3 and 15 there is a complex conjugate couple with negative real part; in rectangle 4 a couple of conjugate purely imaginary roots; in rectangle 5 a conjugate couple with positive real part.

The two branches of the curve $\Delta$, its two asymptotes and the two coordinate axes (we call them "the six sets" for short) divide the plane $O b c$ into 13 domains. Eight of them constitute four couples of domains symmetric w.r.t. the bisector $\{b=c\}$. Consider any smooth arc $V W$ intersecting the six sets as shown on figure 1. Rectangles 1-11 show the evolution of the roots of $P * Q$ along such an arc. Such arcs and their symmetric ones w.r.t. the bisector $\{b=c\}$ intersect 11 out of the 13 domains. Therefore one can understand the evolution of the roots of $P * Q$ (excluding only two of the domains, the ones comprising the square $[0,1] \times[0,1])$ by following the arc $V W$. Similar arcs are shown for similar reasons on figures 3,4 , and 5 .

In rectangle $9 \mathrm{a}$ the two roots are opposite to one another. In rectangle 9 (resp. 9b) the left (resp. the right) of them is closer to 0 .

The numbers of the rectangles are chosen such as to have the same numbers (when no letter "a," "b," "c," or "d" is added) on figure 1 and on figure 3 when the same configuration of roots occurs.

\subsection{A factorization connected with CSS}

Observe that the composition of Schur-Szegö is associative and commutative, i.e., one has $P * Q=Q * P$ and $(P * Q) * R=P *(Q * R)$, so we write simply $P * Q * R$. The following proposition shows that every polynomial having one of its roots at $(-1)$ can be factorized in the sense of CSS into a composition of polynomials which are 
as simple as possible. Lemma A allows one to define a similar factorization for any polynomial different from $x^{n}$.

Proposition 1.1. Every degree $n$ polynomial having one of its roots at $(-1)$ is representable in the form $K_{a_{1}} * \cdots * K_{a_{n-1}}$ where $K_{a}:=(x+1)^{n-1}(x+a)$. This representation is unique modulo permutation of the $a_{i}$.

Proof. One has $K_{a}=\sum_{j=0}^{n}\left(C_{n-1}^{j-1}+C_{n-1}^{j} a\right) x^{j}$. Hence,

$$
K:=K_{a_{1}} * \cdots * K_{a_{n-1}}=\sum_{j=0}^{n} L_{j} x^{j}
$$

where

$$
\begin{aligned}
& L_{j}=\left(\prod_{k=1}^{n-1}\left(C_{n-1}^{j-1}+C_{n-1}^{j} a_{k}\right)\right) /\left(C_{n}^{j}\right)^{n-2}, \quad j<n, \\
& L_{n}=1 .
\end{aligned}
$$

Set $d_{j}:=1 /\left(C_{n}^{j}\right)^{n-2}, \sigma_{l}:=\sum_{\nu_{1}<\cdots<\nu_{l}} a_{\nu_{1}} \cdots a_{\nu_{l}}$. Hence, for $j \geq 1$ one has

$$
L_{j}=d_{j} \sum_{s=0}^{n-1}\left(C_{n-1}^{j}\right)^{s}\left(C_{n-1}^{j-1}\right)^{n-1-s} \sigma_{s} .
$$

Given a monic polynomial $S:=\sum_{j=0}^{n} g_{j} x^{j}, S(-1)=0$, try to find $a_{\nu}, \nu=1, \ldots, n-1$ such that $S=K$, i.e., $L_{j}=g_{j}$ for $j=0, \ldots, n-1$. The determinant of this system of linear equations up to a nonzero factor equals $V:=W\left(\beta_{1}, \ldots, \beta_{n-1}\right)$ where $W$ is the Vandermonde determinant and $\beta_{j}=C_{n-1}^{j} / C_{n-1}^{j-1}=(n-j) / j$. One has $V \neq 0$. Hence, the symmetric functions $\sigma_{j}$ of the numbers $a_{i}$ are uniquely defined and up to permutation, the $a_{i}$ as well.

Remark 1.2. The dependence of the numbers $a_{j}$ on the roots of the polynomial $S$ is not trivial. In particular, the presence of multiple roots in the polynomial to be factorized does not imply that there are equal among the numbers $a_{j}$ and vice versa. Neither does the hyperbolicity of this polynomial imply that all $a_{i}$ are real and vice versa. We illustrate this by the following examples:

- For the polynomial $(x+1) x^{n-1}$ (with an $(n-1)$-fold zero at 0$)$ one has $a_{j}=-\frac{j-1}{n-j+1}, j=1, \ldots, n-1$, i.e., only $a_{1}$ is 0 . Indeed, up to permutation of the indices $j$, the coefficient before $x^{j-1}$ in $K_{a_{j}}$ must equal 0 .

- If $a_{1}=\cdots=a_{n-1}=a>1$, then by [3, Proposition 1.4 and Theorem 1.6] all roots of $K_{a_{1}} * \cdots * K_{a_{n-1}}$ are real and simple. 
- For $n=3$ one has $K_{0}=x^{3}+2 x^{2}+x$. Hence $K_{0} * K_{0}=(x+1)\left(x+\frac{1}{3}\right) x$. For $a, b \in \mathbf{R}$ small enough $K_{a+b i} * K_{a-b i}$ has three distinct negative roots. In this example the two numbers $a_{1}=a-b i, a_{2}=a+b i$ are complex conjugate, no matter that the roots of $K_{a+b i} * K_{a-b i}$ are real and distinct.

- For $n=3$ one has $K_{-2}=x^{3}-3 x-2, K_{-1}=x^{3}+x^{2}-x-1$. Hence, $K_{-2} * K_{-1}=x^{3}+x+2$ which has a single real root $(-1)$, i.e., the composition of two polynomials $K_{a_{1}}, K_{a_{2}}$ with $a_{i}$ real can be a polynomial with a complex conjugate couple.

\subsection{The composition of two polynomials of the form $(x-1)^{n-1}(x+b)$}

Consider the polynomials

$$
S=(x-1)^{n-1}(x+b)=x^{n}+(b-(n-1)) x^{n-1}+\cdots+(-1)^{n-1} b
$$

and

$$
T=(x-1)^{n-1}(x+c)=x^{n}+(c-(n-1)) x^{n-1}+\cdots+(-1)^{n-1} c .
$$

For $n=2$ one has $S=P, T=Q$, where $P$ and $Q$ are defined by (1). It follows from [3, Proposition 1.4] that $S * T$ has an $(n-2)$-fold root at $(-1)$. Hence, one has

$$
S * T=x^{n}+\frac{(b-(n-1))(c-(n-1)) x^{n-1}}{n}+\cdots+b c=(x+1)^{n-2}\left(x^{2}+\alpha x+\beta\right)
$$

where $\alpha=\frac{b c-(n-1)(b+c)+1}{n}, \beta=b c$. The polynomial $x^{2}+\alpha x+\beta$ has a double root if and only if the following condition holds:

$$
\Delta_{n}: \quad F(b, c):=n^{2}\left(\alpha^{2}-4 \beta\right)=(b c-(n-1)(b+c)+1)^{2}-4 n^{2} b c=0 .
$$

We first prove some properties of the curve $\Delta_{n}$, see Lemmas 1.3, 1.4, and 1.7 and Remarks 1.5 and 1.6. After this we give the description of the elements of figure 3 which are new w.r.t. figure 1 .

Lemma 1.3. The curve $\Delta_{n}$ contains no point of the domain $\{b c<0\}$. For $b=0$ (resp. $c=0)$ it contains only the point $\left(0, \frac{1}{n-1}\right)$ (resp. $\left(\frac{1}{n-1}, 0\right)$ ). For $b<0, c<0$, it contains only the point $(-1,-1)$ which is an ordinary double point of $\Delta_{n}$ when considered as a complex curve.

Proof. The first two claims of the lemma follow directly from the formula defining $\Delta_{n}$. To prove the third claim consider the graph of the function $w=F(b, c)$ in the space Obcw. Its critical points are defined by the conditions $\frac{\partial F}{\partial b}=\frac{\partial F}{\partial c}=0$, i.e.,

$$
\begin{aligned}
2(b c-(n-1)(b+c)+1) & (c-(n-1))-4 n^{2} c \\
= & 2(b c-(n-1)(b+c)+1)(b-(n-1))-4 n^{2} b=0 .
\end{aligned}
$$



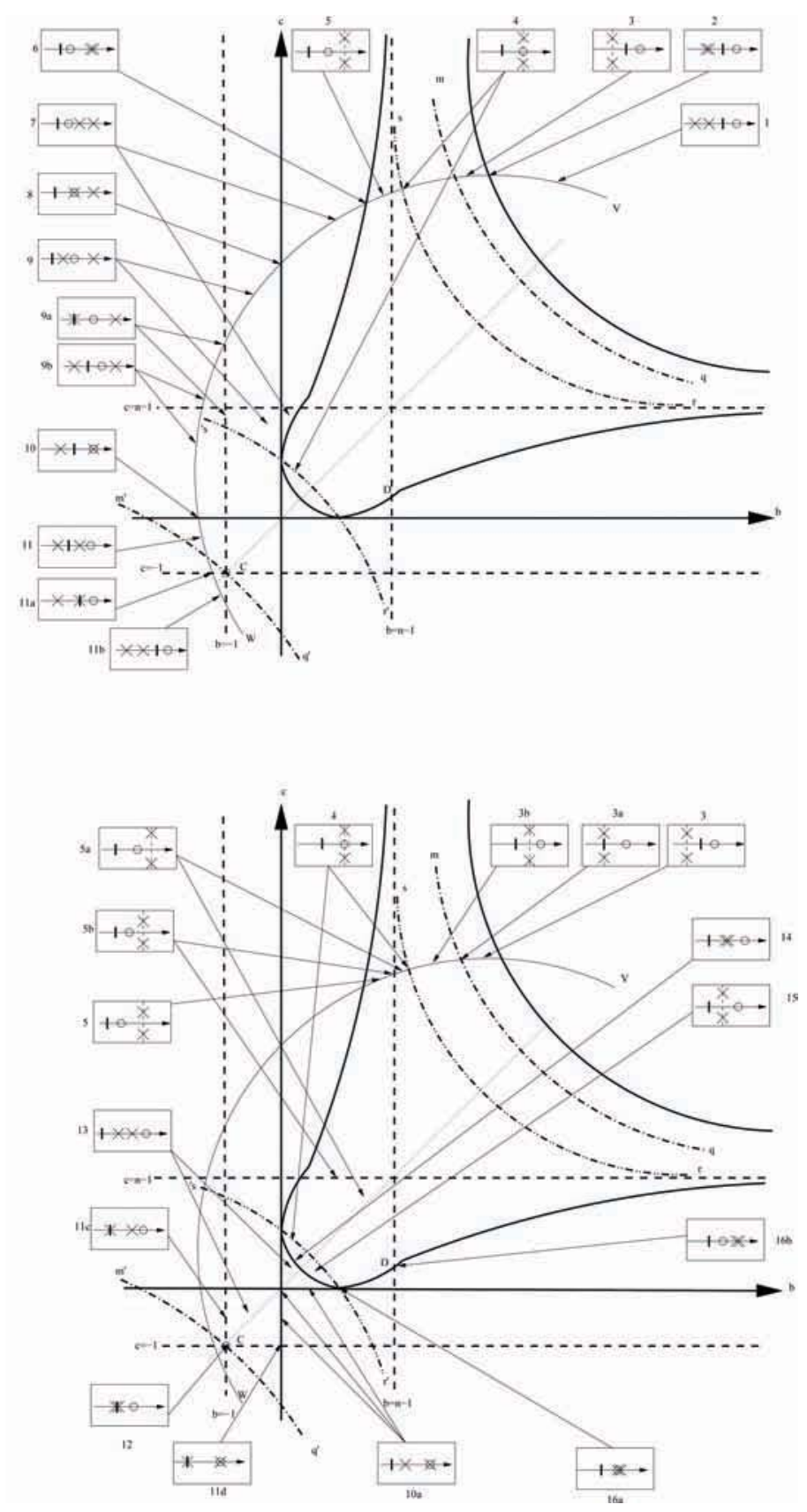

Figure 3 - The curve $\Delta_{n}$. 
For $b<0, c<0$, one has $b c-(n-1)(b+c)+1>0$ and one deduces from (4) that

$$
\frac{b c-(n-1)(b+c)+1}{2 n^{2}}=\frac{b}{b-(n-1)}=\frac{c}{c-(n-1)},
$$

which implies $b=c$. It follows from (4) and $b=c$ that one has

$$
2\left(b^{2}-2(n-1) b+1\right)(b-(n-1))-4 n^{2} b=2(b+1)\left(b^{2}+(2-3 n) b-(n-1)\right)=0,
$$

i.e., either $b=c=-1$ or $b=c=g_{ \pm}$where $g_{ \pm}=\frac{3 n-2 \pm \sqrt{9 n^{2}-8 n}}{2}$.

For $b=c=-1$ the function $F$ has a minimum equal to 0 . Indeed, the Hessian matrix of $F$ equals

$$
H=\left(\begin{array}{cc}
2(c-(n-1))^{2} & 4(b-(n-1))(c-(n-1))-6 n^{2}+4 n \\
4(b-(n-1))(c-(n-1))-6 n^{2}+4 n & 2(b-(n-1))^{2}
\end{array}\right) .
$$

For $b=c=-1$ it has two positive eigenvalues. One has $F\left(g_{-}, g_{-}\right)>F(-1,-1)=0$ because the degree 4 polynomial $\kappa(b):=F(b, b)$ has minima for $b=-1$ and $b=g_{+}$ and a maximum at $b=g_{-}$.

For $r>\frac{2 n^{2}}{n-1}$ consider the quarter of a closed disc $\delta:=\left\{b \leq 0, c \leq 0, b^{2}+c^{2} \leq r^{2}\right\}$. On the arc $\left\{b<0, c<0, b^{2}+c^{2}=r^{2}\right\}$ one has $\alpha^{2}>4 \beta$ because

and

$$
-(n-1)(b+c)=(n-1)(|b|+|c|) \geq(n-1) \sqrt{b^{2}+c^{2}}=(n-1) r
$$

$$
\begin{aligned}
n^{2} \alpha^{2} & =(b c-(n-1)(b+c)+1)^{2} \\
& >2 b c(n-1)(|b|+|c|) \geq 2 b c(n-1) r \geq 4 n^{2} b c=4 n^{2} \beta .
\end{aligned}
$$

Therefore the function $F$ attains its minimal value in $\delta$ either at $(-1,-1)$ or for $b=0$ or for $c=0$. In the last two cases one has $F \geq 0$ with equalities only for $(b, c)=\left(0, \frac{1}{n-1}\right)$ and $(b, c)=\left(\frac{1}{n-1}, 0\right)$.

Hence, for $b<0, c<0$, one has $F \geq 0$ with equality only for $b=c=-1$. The matrix $H$ for $b=c=-1$ being nondegenerate, it is an ordinary double point of $\Delta_{n}$ considered as a complex curve.

Lemma 1.4. (i) The curve $\Delta_{n}$ has asymptotes $\{b=n-1\}$ and $\{c=n-1\}$.

(ii) The curve $\Delta_{n}$ is symmetric w.r.t. the bisector $\{b=c\}$. Its equation is invariant w.r.t. the changes $b \mapsto \frac{1}{b}, c \mapsto \frac{1}{c}$.

(iii) Except the isolated point at $(-1,-1)$, it consists of two branches.

(iv) One of the branches (denoted by $\Delta_{n}^{t}$ ) has ordinary tangencies with the coordinate axes at the points $\left(0, \frac{1}{n-1}\right)$ and $\left(\frac{1}{n-1}, 0\right)$. The other branch is denoted by $\Delta_{n}^{w}$.

(v) Except at these two points there is nowhere a horizontal or a vertical tangent line to the curve. 
(vi) The equations of the two branches of $\Delta_{n}$ are

$$
\Delta_{n}^{t}: \quad b c-(n-1)(b+c)+1=-2 n \sqrt{b c}, \quad \Delta_{n}^{w}: \quad b c-(n-1)(b+c)+1=2 n \sqrt{b c} .
$$

Remark 1.5. Only for $n=2$ do the asymptotes pass through the points of tangency of $\Delta_{n}$ with the coordinate axes.

Proof. $1^{\circ}$. Consider equation (3) as an equation of second degree w.r.t. the variable $b$ and with parameter $c$ :

$$
(c-(n-1))^{2} b^{2}+\left(-4 n^{2} c+2(c-(n-1))(1-(n-1) c)\right) b+(1-(n-1) c)^{2}=0 .
$$

Its discriminant equals $16 n^{2}(n-1) c(c+1)^{2}$. Hence, for $c>0, c \neq n-1$, it has two real distinct roots. For $c=n-1$ the equation is of degree 1 , hence, it has a single real root. For $c=0$ it has a double root $b=\frac{1}{n-1}$. Hence, the curve looks (topologically) as shown on figure 3 (it is drawn by a solid line of width 3 ). This proves part (iii) of the lemma.

$2^{\circ}$. When $b \rightarrow+\infty$, the equation (up to a nonzero factor) tends to

$$
c^{2}-2(n-1) c+(n-1)^{2}=0
$$

and its roots are close to $n-1$. This means that the line $\{c=n-1\}$ is a horizontal asymptote for $\Delta_{n}$.

$3^{\circ}$. The symmetry w.r.t. $\{b=c\}$ (part (ii) of the lemma) follows from the symmetry between $S$ and $T$ in the definition of $S * T$. The invariance w.r.t. the change $b \mapsto \frac{1}{b}, c \mapsto \frac{1}{c}$ is to be checked directly. Hence, $\{b=n-1\}$ is a vertical asymptote for $\Delta_{n}$. Part (i) of the lemma is proved.

$4^{\circ}$. Write equation (5) in the form

$$
A(c) b^{2}+B(c) b+(1-(n-1) c)^{2}=0 .
$$

One has $B_{0}:=B\left(\frac{1}{n-1}\right) \neq 0$, hence, for $(b, c)$ close to $\left(0, \frac{1}{n-1}\right)$ one has

$$
b=-\frac{(1-(n-1) c)^{2}}{B_{0}}+o\left((1-(n-1) c)^{2}\right),
$$

which proves part (iv) of the lemma.

$5^{\circ}$. Consider again (3) as an equation with unknown variable $b$. When simple, its roots depend smoothly on the parameter $c$. Therefore there is no vertical tangent line to $\Delta_{n}$ except at the point $\left(0, \frac{1}{n-1}\right)$. Using part (ii) of the lemma, one deduces that there is no horizontal tangent line to $\Delta_{n}$ except at the point $\left(\frac{1}{n-1}, 0\right)$. This proves part (v).

$6^{\circ}$. It follows from the equation of $\Delta_{n}$ that the equations of its two branches are (up to permutation) as in (vi) of the lemma. To prove part (vi) it suffices to justify the minus sign in the right hand-side of $\Delta_{n}^{t}$. Write the left hand-side of the equation 
of the branch in the form $c(b-(n-1))-(n-1) b+1$. When the point of $\Delta_{n}^{t}$ is close to the asymptote $\{b=n-1\}$, one has $c>0$ and $b<n-1, b \approx n-1$, therefore one has $c(b-(n-1))<0$ and (for $n>2)-(n-1) b+1<0$; for $n=2$ the left-hand side of the equation of the branch $\Delta_{n}^{t}$ reduces to $(b-1)(c-1)$ which is negative for large $c$ and $b \approx 1, b<1$.

Remark 1.6. The form of the curve $\Delta_{n}$, see figure 3 , can be deduced from the above lemma. Indeed, consider the intersections of $\Delta_{n}$ with the lines $\left\{b=b_{0}\right\}, b_{0} \geq 0$. For $b_{0}=0$ the intersection is the point $\left(0, \frac{1}{n-1}\right)$. For $b_{0} \in(0, n-1)$ the intersection consists of two points, the $c$-coordinate of the "above" point is increasing, the $c$-coordinate of the point "below" is decreasing on $\left(0, \frac{1}{n-1}\right]$ and then increasing on $\left[\frac{1}{n-1}, 0\right)$. This follows from parts (iv) and (v) of the lemma. For $b_{0} \in(n-1,+\infty)$ the $c$-coordinate of the point "below" is increasing and as it tends to the asymptote $\{c=n-1\}$ when $b \rightarrow+\infty$, it must tend to it "from below". For $b_{0}>n-1$ and close to $n-1$ the point "above" has a large positive $c$-coordinate. For $b \rightarrow+\infty$ it must tend to the same asymptote. The absence of horizontal tangent lines implies that it tends to it "from above." This justifies the form of the curve as presented on figure 3 except the possible presence of more than two inflection points. In particular, it justifies the fact that each line $\left\{b=b_{0}\right\}, b_{0}>0, b_{0} \neq n-1$, and each line $\left\{c=c_{0}\right\}, c_{0}>0, c_{0} \neq n-1$, intersects the curve at exactly two points.

Lemma 1.7. The curve $\Delta_{n}$ has exactly two inflection points as shown on figure 3 .

Proof. $1^{\circ}$. The curve $\Delta_{n}$ is defined by an equation of degree 4 . Therefore the curve can have an order of contact at most 4 with any line. We call ordinary (resp. degenerate) inflection point one at which the order of contact of $\Delta_{n}$ with the tangent line at the given point is equal to 3 (resp. to 4 ).

Recall that the branches $\Delta_{n}^{t}$ and $\Delta_{n}^{w}$ of $\Delta_{n}$ were defined in Lemma 1.4. If the curve $\Delta_{n}$ has more than two ordinary inflection points, then at least one of the three situations takes place:

- there are at least two ordinary inflection points on $\Delta_{n}^{w}$ (their number is even due to the symmetry of $\Delta_{n}$ w.r.t. the bisector $\left.\{b=c\}\right)$;

- there are three or more ordinary inflection points on $\Delta_{n}^{t}$ to the right of the point $\left(\frac{1}{n-1}, 0\right)$ and the same number (due to the same symmetry) of inflection points above the point $\left(0, \frac{1}{n-1}\right)$; the number of inflection points to the right of $\left(\frac{1}{n-1}, 0\right)$ is odd because $\Delta_{n}^{t}$ approaches the asymptote $\{c=n-1\}$ from below, see the above remarks;

- there are at least two ordinary inflection points on $\Delta_{n}^{t}$ between the two points of tangency (i.e., between $\left(0, \frac{1}{n-1}\right)$ and $\left.\left(\frac{1}{n-1}, 0\right)\right)$.

In all three cases the presence of so many ordinary inflection points implies that there exists a line $\theta: c=\alpha b+\varepsilon, \theta \subset O b c$, with two points of tangency with $\Delta_{n}$. By part (v) of Lemma 1.4, this line cannot be vertical or horizontal. 
We prove that the following situation is also impossible:

- the curve $\Delta_{n}$ has a degenerate inflection point.

$2^{\circ}$. Suppose that one of the four situations from $1^{\circ}$ takes place. Set $c=\lambda b+\varepsilon$ in equation $(3)$ :

$$
(b(\lambda b+\varepsilon)-(n-1)(b+\lambda b+\varepsilon)+1)^{2}-4 n^{2} b(\lambda b+\varepsilon)=0 .
$$

Its left hand-side is of the form

$$
\left(\lambda b^{2}+A b+B\right)^{2}-4 n^{2} b(\lambda b+\varepsilon), \quad A=\varepsilon-(n-1)(1+\lambda)
$$

and it must be also of the form

$$
\left(\lambda b^{2}+A b+C\right)^{2}
$$

(it is the same $A$; this coefficient is defined by the monomials in $b$ of degree 3 and 4 ). Form $(*)$ results from the fact that there are either two points of tangency or a degenerate inflection point. Hence,

$4 n^{2} b(\lambda b+\varepsilon)=\left(\lambda b^{2}+A b+B\right)^{2}-\left(\lambda b^{2}+A b+C\right)^{2}=(B-C)\left(2 \lambda b^{2}+2 A b+B+C\right)$.

This means that one has $B+C=0$ and $A=\varepsilon$. The last equality and $A=\varepsilon-$ $(n-1)(1+\lambda)$ imply $\lambda=-1$.

$3^{\circ}$. Set $\varphi:=b(-b+\varepsilon)$. Write equation (6) in the form

$$
(\varphi-(n-1) \varepsilon+1)^{2}-4 n^{2} \varphi=0 .
$$

It has two double roots when considered as an equation with unknown variable $b$, so these roots satisfy also the condition

$$
Z \varphi^{\prime}=0 \quad \text { where } \quad Z(\varphi)=2(\varphi-(n-1) \varepsilon+1)-4 n^{2} .
$$

If one has $Z=0$ and ( 7$)$, then one has $\varphi=n^{2}$ and $\varepsilon=-n-1$, i.e., $b$ satisfies the equation $b^{2}+(n+1) b+n^{2}=0$ which has no positive root.

If one has $\varphi^{\prime}=0$ and $(7)$, this implies $b=\frac{\varepsilon}{2}, \varphi=\frac{\varepsilon^{2}}{4}$, and

$$
\left(\frac{\varepsilon^{2}}{4}-(2 n-1) \varepsilon+1\right)\left(\frac{\varepsilon^{2}}{4}+\varepsilon+1\right)=0
$$

whose roots are $\varepsilon_{ \pm}:=2\left(2 n-1 \pm \sqrt{4 n^{2}-4 n}\right),-2,-2$.

$4^{\mathrm{o}}$. For $\varepsilon=-2$ one obtains the line $c=-b-2$ passing through the isolated point $(-1,-1)$, for the other two values one obtains the tangent lines to $\Delta_{n}$ passing through its intersection points with the bisector $\{b=c\}$. 
For $\varepsilon=\varepsilon_{ \pm}$the line $\theta$ has a contact of order 2 with $\Delta_{n}$. Indeed, if the order is greater than or equal to 3 , then $b$ must satisfy equations $(7),(8)$, and

$$
Z^{\prime} \varphi^{\prime}+Z \varphi^{\prime \prime}=0
$$

As we saw in $3^{\circ}$, one has $\varphi^{\prime}=0 \neq Z$. Therefore equation $(* *)$ implies $\varphi^{\prime \prime}=0$. However, one has $\varphi^{\prime \prime}=-2$ which is a contradiction.

For $\varepsilon=\varepsilon_{ \pm}$there is just one point of tangency between $\Delta_{n}$ and $\theta$. Indeed, the symmetry of $\Delta_{n}$ w.r.t. $\{b=c\}$ implies that if there is more than one such point, then there should be at least three of them. This means that equation (7) should be of degree at least 6 w.r.t. $b$ which is false.

It is impossible to have two, four, six, etc., inflection points between the points $\left(\frac{1}{n-1}, 0\right)$ and $\left(0, \frac{1}{n-1}\right)$ or on the branch $\Delta_{n}^{w}$. Indeed, this would mean that there are more than three distinct values of $\varepsilon$ for which there is a line $\theta: c=-b+\varepsilon$ tangent to $\Delta_{n}$ which is also false.

Hence, neither of the four possibilities mentioned in $1^{\circ}$ is realized, i.e., $\Delta_{n}$ has only two inflection points as shown on figure 3 ; they are ordinary ones.

On figure 3 there are more rectangles than on figure 1 showing different root configurations because we take into account also the position of $(-1)$ which is a root of $S * T$. The latter is represented in the rectangles by a small vertical line. The great number of rectangles is the reason why we separate them in the two different pictures comprising figure 3. Some of the rectangles are given in both pictures for the reader's convenience. The reader can compare the evolution of the roots given by rectangles 1-11 on figure 1 and by the ones on the first picture of figure 3 .

In rectangles $5 \mathrm{a}, 5 \mathrm{~b}$, and 5 the real part of the complex conjugate couple is respectively smaller than, equal to, and bigger than 1 .

On the hyperbola

$$
H_{0}:(b-(n-1))(c-(n-1))=n(n-2)
$$

the conjugate couples have zero real part. Indeed, the coefficient before $x^{n-1}$ of $S * T$ (see $(2)$ ) equals the minus sum of the roots of $S * T$; these roots include the $(n-2)$-fold root at $(-1)$, the remaining two are not real, hence, they are conjugate and their real part is 0 . This hyperbola is denoted by $s-r$ and $s^{\prime}-r^{\prime}$ on figure 3 . It contains the two points of tangency $\left(\frac{1}{n-1}, 0\right)$ and $\left(0, \frac{1}{n-1}\right)$ of $\Delta_{n}$ with the coordinate axes. Notice that for $n=2$ the equation of $H_{0}$ defines not a hyperbola, but the couple of asymptotes $\{b=1\}$ and $\{c=1\}$.

On one of the branches of the hyperbola

$$
H_{-1}:(b-(n-1))(c-(n-1))=n^{2}
$$

the conjugate couples have real part equal to $(-1)$. The reasoning is similar to the one concerning $H_{0}$. This hyperbola is denoted by $m-q$ and $m^{\prime}-q^{\prime}$ on figure 3 , 


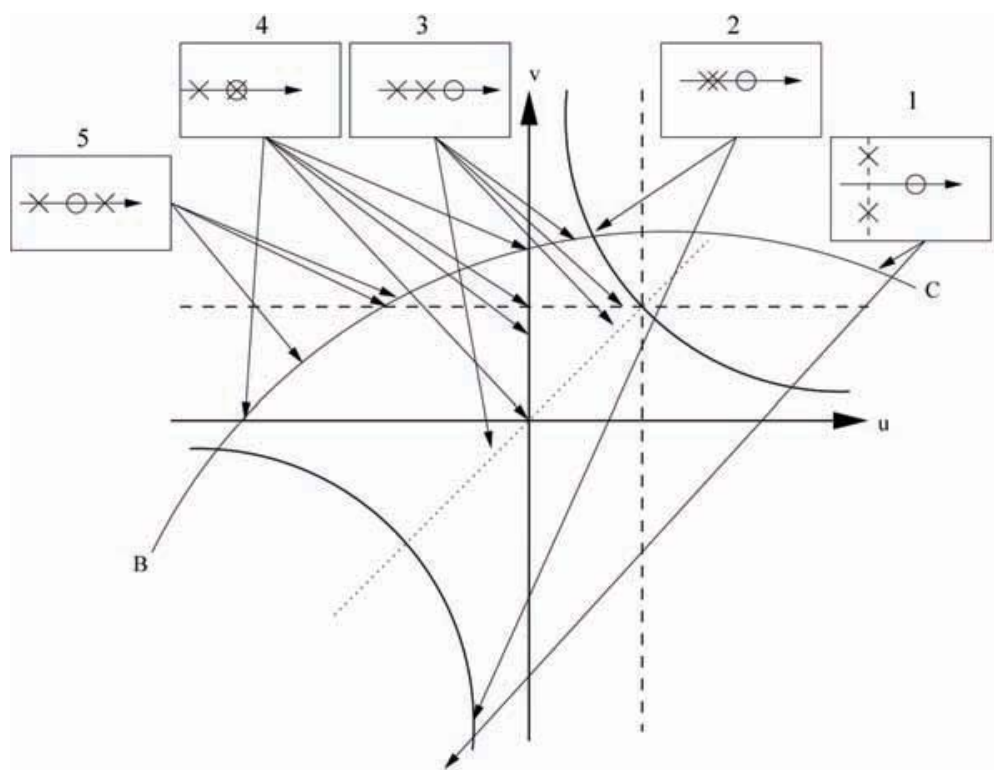

Figure 4 - The case of real polynomials of degree 2 .

and the branch in question is $m-q$. On the other branch of $H_{-1}$ the two roots of $S * T$ different from $(-1)$ are real and symmetric w.r.t. $(-1)$. This branch contains the isolated point $(-1,-1)$. On figure 1 the hyperbola $H_{-1}$ is absent because for $n=2$ there is no point in comparing the roots of $P * Q$ with $(-1)$.

The two hyperbolas share the same asymptotes with $\Delta_{n}(\{b=n-1\}$ and $\{c=n-1\})$. These hyperbolas and the branch $\Delta_{n}^{w}$ of $\Delta_{n}$ are positioned as shown on figure 3. This follows from the equations of the hyperbolas and from the equation of the branch $\Delta_{n}^{w}$ presented in the form $(b-(n-1))(c-(n-1))=n(n-2)+2 n \sqrt{b c}$, see part (vi) of Lemma 1.4. Indeed, for the right hand-sides one has $n(n-2)+2 n \sqrt{b c}>$ $n^{2}>n(n-2)$; the left inequality follows from $b>n-1$ and $c>n-1$ on $\Delta_{n}^{w}$.

\subsection{The case of real polynomials}

Consider the situation when $P$ and $Q$ are real. Consider first the generic situation when $a_{1} \neq 0 \neq b_{1}$. Using Lemma A we assume that $P=x^{2}+x+u, Q=x^{2}+x+v$. Hence, $P * Q=x^{2}+\frac{x}{2}+u v$. This polynomial has a double root if and only if one has

$$
\chi: 16 u v=1 .
$$

On figure 4 we present the hyperbola $\chi$ and (in the same way as on figure 1) the roots of $P * Q$ in small rectangles. The root configuration is the same at $(u, v)$ and 
at $(v, u)$ because $P$ and $Q$ play the same roles. It is also the same at $(u, v)$ and at $(-u,-v)$ because $P * Q$ is the same. The evolution of the roots of $P * Q$ can be understood if one follows the $\operatorname{arc} C B$.

The polynomials $P$ and $Q$ are hyperbolic respectively for $u \leq \frac{1}{4}$ and $v \leq \frac{1}{4}$. The lines $u=\frac{1}{4}$ and $v=\frac{1}{4}$ (where these polynomials have double roots at $\left(-\frac{1}{2}\right)$ ) are drawn by dashed lines. The root configuration of $P * Q$ does not change when one of these lines is crossed.

In the case when $a_{1} b_{1}=0$ one can set $P=x^{2}+2 a_{1} x+u, Q=x^{2}+2 b_{1} x+v$, hence, $P * Q=x^{2}+u v$. For $u v<0$ this polynomial has two distinct and opposite real roots $\pm \sqrt{u v}$, for $u v=0$ it has a double zero root, and for $u v>0$ it has a couple of conjugate imaginary roots $\pm i \sqrt{-u v}$. The reader can readily draw a figure similar to (and simpler than) figure 4 which illustrates this case.

\subsection{The mixed case}

Consider the families of polynomials $P=x^{2}+x+b$ and $Q=(x-1)(x+c)=$ $x^{2}+(c-1) x-c$. The first of them is parameterized by its coefficient $b$, the second by its root $(-c)$. This is the generic situation when the polynomial $P$ has a nonzero coefficient before $x$. The above parameterization excludes the possibility $P$ to have a double root at 0 (in which case one has $P * Q=x^{2}$ ). In the generic case the polynomials $P$ and $Q$ can be given the above form thanks to Lemma A. if

One has $P * Q=x^{2}+\frac{(c-1) x}{2}-b c$. This polynomial has a double root if and only

$$
\tilde{\Delta}:=c(16 b+c-2)=-1 .
$$

This equation defines a hyperbola tangent to the $c$-axis at $(0,1)$. (Indeed, for $b=0$ equation (9) has a double root $c=1$.) The hyperbola has asymptotes $\{c=0\}$ and $\{16 b+c=2\}$. The dependence of the roots of $P * Q$ on $b$ and $c$ is shown on figure 5 .

If the polynomial $P$ is of the form $x^{2}+b$ (this is the nongeneric case), then one has $P * Q=x^{2}-b c$ and the study of this case is analogous to the one of the degenerate case $a_{1} b_{1}=0$ considered at the end of subsection 1.4 .

\section{References}

[1] T. Craven and G. Csordas, Multiplier sequences for fields, Illinois J. Math. 21 (1977), no. 4, 801-817.

[2] Composition theorems, multiplier sequences and complex zero decreasing sequences, Value distribution theory and related topics (G. Barsegian, I. Laine, and C. C. Yand, eds.), Adv. Complex Anal. Appl., vol. 3, Kluwer Acad. Publ., Boston, MA, 2004, pp. 131-166.

[3] V. Kostov and B. Shapiro, On the Schur-Szegö composition of polynomials, C. R. Math. Acad. Sci. Paris 343 (2006), no. 2, 81-86.

[4] V. V. Prasolov, Polynomials, Algorithms and Computation in Mathematics, vol. 11, SpringerVerlag, Berlin, 2004. Translated from the 2001 Russian second edition by Dimitry Leites. 


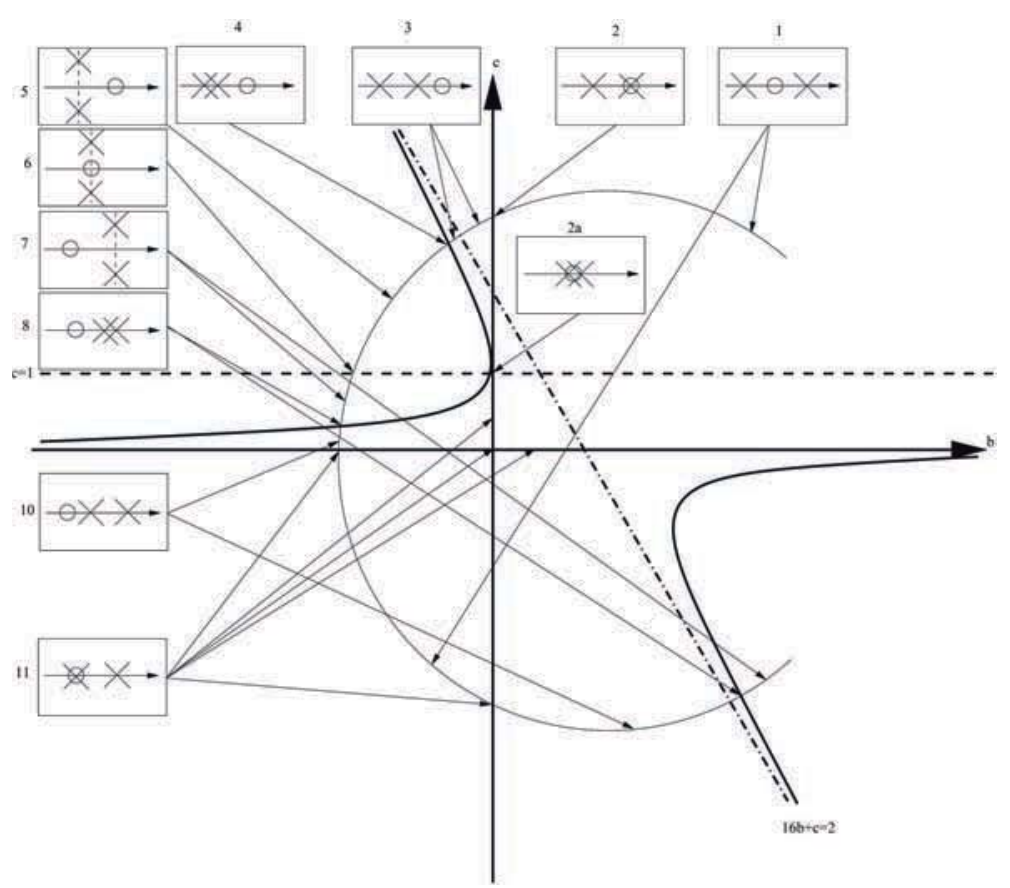

Figure 5 - The mixed case in degree 2 .

[5] Q. I. Rahman and G. Schmeisser, Analytic theory of polynomials, London Mathematical Society Monographs. New Series, vol. 26, The Clarendon Press Oxford University Press, Oxford, 2002. 